\title{
Field Museum of Natural History.
}

Publication 187.

Botanical Series.

Vol. II, No. II.

\section{CONTRIBUTIONS TO NORTH AMERICAN EUPHORBIACEAE-VI}

\section{VEGETATION OF ALACRAN REEF}

BY

Charles Frederick Millspaugh

Curator Department of Botany.

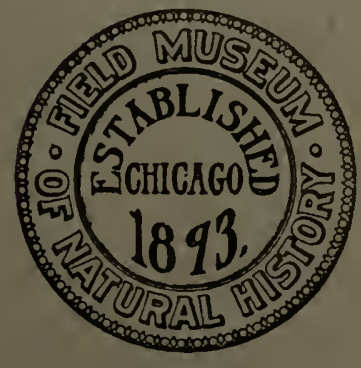

Chicago, U. S. A.

April, IgI6. 

Field Museum of Natural History.

Publication 187.

Botanical Series.

Vol. II, No. II.

\section{CONTRIBUTIONS TO NORTH AMERICAN EUPHORBIACEAE-VI}

\section{VEGETATION OF ALACRAN REEF}

BY

Charles Frederick Millspaugh

Curator Department of Botany.

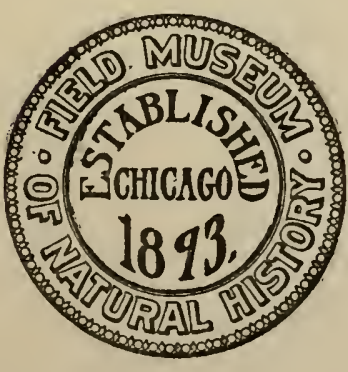

Chicago, U.S. A. April, 1916. 



\title{
CONTRIBUTIONS TO NORTH AMERICAN EUPHORBIACEÆ-VI
}

\author{
CHARLES F. MILLSPAUGH, M. D.
}

Chamaesyce S. F. Gray, Nat. Arr. Brit. P1. 2:260. I82I.

Leaves opposite, inaequilateral; inflorescence solitary or capitulate, axillary and terminal; bracteoles plumose or ciliate; seeds minute, ovoid or elongate-ovoid, more or less quadrangular the facets smooth or transverse-rugulose.

Type species: Euphorbia peplis Linn. Sp. P1. 652. I753.

\section{Chamaesyce barbicarina sp. nov.}

Plants semi-erect, about $2 \mathrm{dm}$. high, annual. Stems many, from a but slightly raised rootstalk; branches many, virgate, terete, long-pilose especially upon the upper surface. Inflorescence solitary in the axils of the terminal branchlet leaves. Leaves opposite, $5-8 \times 2-4 \mathrm{~mm}$., ovate, obtuse, broadest at the rounded apex, base strongly auriculooblique, glabrous the thickened margin crenate-dentate; petioles about Imm., hairy; stipules broadly triangular-lanceolate, lacerate. Involucres turbinate, glabrous without and within; peduncle about the length of the involucre (in fruit twice the length); involucral lobes triangular, ciliate; sulcus shallow; glands scutelliform; appendages white, irregular-ovate, margin crenate or 2-3-crenate-dentate; bracteoles none; male pedicels few, short; female pedicel ciliate-hairy; styles short, bilobed at the apex. Capsule obpyriform, glabrous except on the blunt keels of the otherwise smooth carpels which are long-ciliate fringed; seeds red, ovoid, acute, $.9 \times .6 \mathrm{~mm}$., angles sharp, facets marked by a few very slightly raised irregular and broken transverse ridges.

Between $\mathrm{Ch}$. dioica and $\mathrm{Ch}$. yucatanensis but readily distinguished from both by the peculiar ciliate-fringed keels of the carpels.

Sitilpech Road, November, 1895, Gaumer 939a (Type sheet, Field Mus. cat. no. $196,35^{2}$.) It was gathered as a part of a mass of $\mathrm{Ch}$. rutilis by the collector. Also collected at Chichankanab, Gaumer 1452, 2074; and at Pocoboch 2405. 
Chamaesyce cozumelensis comb. nov.

Euphorbia cozumelensis Millsp. Field. Mus. Bot. 2:26I. I900.

A large number of sheets of this species show an intergradation from the absolutely glabrous and glaucous original collection, on Cozumel Island, through a form with a few long, scattered hairs in the capsular sulci ( $E$. cozumelensis pilosulca Millsp. ibid. 262); a form with a few long hairs on the nodes, petioles and leaves; to plants densely long-pilose throughout. The extremes of these plants appear like widely different species but the characters do not warrant the application of even varietal names.

The distribution of the forms is as follows: The glaucous form Cozumel Island, Millspaugh I604, I606, I608, I609, I6II; the form with the capsular sulci hairy - Cozumel Island, Millspaugh 1501, 1502; the form with the nodes, petioles and leaves long-pilose - Cozumel Island, Millspaugh 49, 53, Chichankanab, Gaumer 1456; whole plant densely long-pilose - Mugeres Island, Millspaugh 24, Izamal, Silam and Progreso, Gaumer 314, 629, 1887, 2407.

Chamaesyce hirta (L.) Millsp. Field Mus. Bot. 2:303.

I have gathered a large number of forms of this species from many localities in Hawaii, south China, Straits Settlements, Java, the Philippines, Burma, India, and from Bermuda, Florida and the Bahamas through the West Indian islands, Mexico and South America to Pernambuco, Brazil. With all this material and a large number of sheets from other collectors I can not satisfy myself that there is any specific differentiation between $\mathrm{E}$. hirta and E. pilulifera L., capitata Lam., globulifera Kth., verticillata Vell., nodiflora Steud., procumbens DC., obliterata Jacq., ophthalmica Pers., discolor Engelm., gemella Lag., and Karwinskyi Boiss. the type material of all of which I have examined. These are only intergradient forms and there are a multitude of others that have luckily escaped specific nomination.

Chamaesyce Rosei sp. nov.

Annual, short-crispid-multicellular hairy. Root straight and deep; stems many, terete, wiry; internodes long; branches profuse, ascending. Inflorescence in terminal, globose heads. Leaves inaequilateral, narrowly lanceolate, $\mathrm{I}^{-25} \times 3^{-6 \mathrm{~mm}}$., crisp-hairy on both surfaces and with a broad, deep-red, central line of maculation; apex acute; base narrowing to the petiole; margin shallow-toothed toward the apex; petioles short (about $2 \mathrm{~mm}$.); stipules mere aristate teeth about $.8 \mathrm{~mm}$. long. Involucres turbinate, roseate, sessile or nearly so, thin walled, the outer surface pubescent the inner densely bearded; lobes deltoid, 
aristate; sulcus $\mathrm{V}$-shaped, the fundus occupied by 2-3-aristate lobes; glands minute, stipitate, cupulate; appendages roseate, distant from the glands and but little larger, inserted about half way down the stipe; male flowers about 6, short, absent in many involucres of each cluster; filaments glabrous; female flower short pedicelled; ovary densely pubescent; styles roseate, bifurcate two-thirds their length, the branches divaricate and somewhat capitulate at the stigmatic tips. Capsule strongly tricoccous, bearing very short, thick, crispid, golden, multicellular hairs; seeds pink-ashen, ovate-quandrangular, acute, $.8 \times .5 \mathrm{~mm}$., angles sharp, facets marked by irregular, complete and incomplete transverse ridges.

Near Ch. hirta (L.) Millsp.

Mexico, along an arroyo in the vicinity of Alamos, State of Sonora, Rose, Standley \& Russell, March I3, r9ro, I2728 (Type in herb. New York Botanical Garden).

\section{Chamaesyce vallis-mortae sp. nov.}

Perennial from a long, cylindrical root; densely woolly-pubescent above. Stems many, wiry, divergently branching toward the tips. Inflorescence solitary in the leaf axils of the branchlets. Leaves thick, ovate, cordate, rounded at the apex, entire, $5 \times 4-6 \times 5 \mathrm{~mm}$., densely woolly on both surfaces; petioles about Imm. long; stipules short, triangular, ciliate. Involucres campanulate; peduncle about one-third the length of the tube; outer surface woolly, inner surface fasciculatehairy; lobes triangular; sulcus deeply $\mathrm{V}$-shaped, the fundus unoccupied; glands green, longitudinal, scutelliform; appendages large, white, flabelliform, the outer margin papulo-ciliate and often slighty 3 -crenate; male flowers about 6 , glabrous; female flower pedicellate; ovary woollypubescent; styles bifid two-thirds their length, the branches divergent not swollen at the stigmatic tips. Capsule obpyriform, hairy; seeds salmon-color, quadrangular-ovate, pointed, the angles rounded, facets smooth or nearly so.

Near Ch. tonsita Millsp.

California, Death Valley between Mohave and Keeler, alt. $75^{\circ}$ meters, June 21, r891, Coville \&o Funston I008 (Type in U. S. National Herbarium cat. no. 16203).

\section{Chamaesyce tamaulipasana sp. nov.}

Annual, prostrate, pilose. Stems many from the thickened rootstalk; branches unilateral on the stems, densely foliate; branchlets very short. Inflorescence solitary in the axils of the branchlet leaves. Leaves appressed, inaequilateral, ovate, acute, cordate, entire, $3.5 \times 2-5 \times 3$ 


\section{Field Museum of Natural History - Botany, Vol. II}

mm., long-crisp-hairy on both surfaces; petioles short, thick; stipules elongate-linear, hairy. Involucres elongate-turbinate narrowing to the peduncle which is about the length of the tube; both surfaces pilose; sulcus U-shaped with a short, linear lobe in the fundus; glands transverse, ovoid, short stipitate, scutelliform; appendages flabelliform, twice the width of the glands, entire, the margin papilliform-ciliate; male flowers few; pedicels glabrous; filaments very short; female flower pedicellate; ovary densely pilose; styles bifurcate to the middle, the stigmatic lobes divergent. Capsule obpyriform, pilose, strongly tricoccous; seeds red-ashen, elongated ovoid-quadrangular, pointed, $\mathrm{r.2} \times$ $.5 \mathrm{~mm}$., angles sharp; facets nearly smooth, indistinctly marked by a median longitudinal line approached on both sides by numerous, irregular, faint, transverse ridges.

$\mathrm{Near} \mathrm{Ch}$. velleriflora (Boiss).

Known only from the vicinity of Victoria, Tamaulipas, Mexico, where it was collected by Dr. Edward Palmer, Feb. I-Apr. 9, 1907 as his no. 98 (Type in herb. Field Museum cat. no. 2I 7,435).

\section{Chamaesyce Standleyi sp. nov.}

Annual, prostrate, finely pilose. Stems many, from a thickened rootstalk; branches densely pilose, short, each subtended by a pair of large leaves; branchlets very short. Inflorescence solitary in the axils of the branchlet leaves. Leaves strongly inaequilateral, ovate, $3 \times 4-5 \times$ ro $\mathrm{mm}$., nearly glabrous, cordate, blunt, lightly crenate-dentate more pronounced at the rounded apex; petioles short $(.8 \mathrm{~mm}$.); stipules linear entire. Involucres cylindro-turbinate on a very short peduncle with bract-like leaflets at the base, densely pilose without and within; lobes triangular, long-ciliate; sulcus narrow, about half the depth of the involucral tube, fundus unoccupied; glands transverse, narrowly scutelliform and somewhat plicate; appendages white, rounded, entire, very narrow on the lower and somewhat broader on the upper pair of glands; male flowers few, very short pedicelled and inserted in a ring at the base of the tube, not as long as the pedicel of the female flower; glabrous; female flower short pedicelled; ovary obpyriform, pilose; styles long, bifurcate at the apex. Capsule pilose, deeply tricoccous; seeds ashen, ovoid-quadrangular, blunt, .9 x. $4 \mathrm{~mm}$., the angles sharp and somewhat included by the ridges; facets marked by $3-4$ often incomplete transverse ridges.

Near Ch. thymifolia (Burm.).

Only known from the vicinity of Acaponeta, Tepic, Mexico, where it was collected by Rose, Standley \& Rose, Apr. 10, I910, no. 14342 (Type in U. S. National Herbarium cat. no. $637,2 \mathrm{Ir}$ ). 
Chamaesyce sulfurea sp. nov.

Annual, prostrate-rosulate, sulphur color when dried, glabrous. Root short; stems many, radiating from the rootstalk, thick, terete; branches and branchlets many, dichotomous. Inflorescence solitary in the leaf axils throughout the plant. Leaves ovate-lanceolate, inaequilateral, somewhat falcate, $5 \times 3-I$ I $7 \mathrm{~mm}$., the midrib prominent beneath, base unequally-cordate, apex acute, margin entire, somewhat thickened and revolute; petioles about one-quarter the length of the blade; stipules lacerate-fimbriate. Involucres globular, 5-columnar, glabrous; peduncle two-thirds the length of the tube; involucral lobes deltoid, truncate, 3-toothed at the apex; sulcus shallow, rounded, unoccupied; glands large, stipitate, scutelliform; appendages none; bracteoles numerous, ligulate, interspersed with 5 , thin, lanceolate, fimbriate, ciliate petaloid rudiments; stamens few, filaments short, glabrous; female flower long-columnar-pedicellate, glabrous; ovary smooth, styles clustered in an erect close mass nearly as large as the ovary itself, styles thick, clavellate, approximate, bifurcate to the middle. Capsule strongly tricoccous, the carpels smooth, line of dehiscence strongly marked; seeds white, I. $5 \times 1 \mathrm{~mm}$., dorsal angle prominent, the whole surface beautifully sculptured into short, rounded, high, broken transverse ridges appearing like a miniature white cluster of earthworm casts.

Near Ch. ocellata from which the seeds readily differentiate it.

Type station Butte Co., California, on hills east of Chico, near Big Chico Creek, September r6, I913, A. A. Heller III40 (Type in herb. Field Museum cat. no. 4II,4II). Also collected at or near the type station by $H$. M. Hall, July, I914, 9759.

\section{Chamaesyce Gooddingii sp. nov.}

Glabrous, prostrate, annual, reddish-green in general color, with a mass of wiry stems and branches from the somewhat thickened rootstalk. Inflorescence solitary in the terminal axils. Leaves ovate, obtuse, $4 \times 2-7 \times 5 \mathrm{~mm}$. palmately 3 -nerved, the midvein prominent beneath, unequally cordate, margin entire; petioles about one-quarter the length of the blade; stipules: the lower filiform, entire, the upper ligulate and often few-lacerate at the apex. Involucres turbinate, glabrous, peduncles about one-half the length of the tube; lobes triangular, apiculate, the margins blunt-ciliate; sulcus shallow, its fundus occupied by a narrow, lanceolate fifth lobe; glands stipitate, transversely narrow-scutelliform, the outer edge crenate-dentate to the appearance of an appendage; bracteoles none; petaloid rudiments flabelliform, manyfimbriate; stamens few, filaments long, glabrous; female flowers longpedicelled, glabrous, styles divergent, bilobate at the apex. Capsule 
glabrous, strongly tricoccous, the carpels rounded-keeled and minutely white-punctate; seeds white, $.9 \times .5 \mathrm{~mm}$., elongated ovoid-quandrangular, angles rounded, the dorsal prominent; facets marked medially with two longitudinal lines of short, rounded, inconspicuous, irregular, transverse rugae.

Near Ch. sulfurea from which its seed readily differentiate it.

Type locality Lee Canyon, Charleston Mountains, in limestone at 8000 ft. alt., Clark Co., Nevada, A. A. Heller II058 (Type in herb. Field Museum cat. no. 4Ir,087); previously gathered by Leslie $N$. Goodding, in sandy washes at Las Vegas, Nevada, in 1907, No. 2282.

\section{Chamaesyce aureola sp. nov.}

An erect, or prostrate, pilose annual (?) with a ligneous root from which spreads a dense mass of terete, divaricate branches and branchlets. Inflorescence solitary, terminal and upperaxillary. Leaves thick. strongly inaequilateral, ovate, obtuse, $2-4 \times 1.5^{-2} \mathrm{~mm}$., densely pilose, cordate, entire; petioles less than one-half the length of the blade; stipules: the lower deltoid, the upper filiform, those of the upper surface with a flat, purplish gland at the base. Involucres turbinate, the throat densely pilose; peduncle about one-half the length of the tube; tube densely pilose without and within; lobes deltoid, pilose; sulcus V-shaped; glands transverse, oblong, reddish-brown, flat and but little cupped; appendages large, white or creamy, ovate, nearly entire; bracteoles many, ligulate, glabrous; stamens few, filaments glabrous, the joint close to the anther base; female pedicel thick, pilose; ovary pilose; styles 3 , bifurcate to the middle, the branches divaricate and not sensibly enlarged at their stigmatic apices. Capsule strongly tricoccous, densely pilose; seeds smooth, white, creamy-white or sage-color, elongate-ovate, I. $2 \times .6 \mathrm{~mm}$., the dorsal angle prominent, 4-angled at the base, pointed at the apex, the facets smooth and showing but a few slight indications of scattered pits.

Near Ch. polycarpa and $\mathrm{Ch}$. vestita from each of which the characters readily distinguish it.

So far seen only in a series of specimens collected at Azusa, Los Angeles Co., California, at an elevation of $800 \mathrm{ft}$. Huron H. Smith 4933 (Type in herb. Field Museum cat. no. 389,282).

Chamaesyce prostrata (Ait.) Small Fl. SE. U. S. 7I3, I333. 1903.

A species of wide distribution misinterpreted in previous Yucatan publications of species as E. rhytisperma and serpyllifolia on account of variation from the type form.

The species is represented in the Yucatan Flora by Schott 967 from Merida where it was gathered in a mass of Ch. hirta; Millspaugh 27 
(I887), also from Merida; Gaumer 254 from cultivated ground at Izamal, 460 from stone walls at the same place, and on waste ground at Tekax, II 23.

Chamaesyce rutilis Millsp. Field Mus. Bot. 2:385. I9I4.

This species of Guatemala and Honduras has turned up in the Yucatan region. The type is Chas. C. Deam, 6189, Field Mus. cat. no. 285 , I I I from Fiscal, Guatemala, alt. $3700 \mathrm{ft}$.

The Yucatan localities are: Chaltumha Road, Sitilpech Road, Gaumer 938, 939.

Chamaesyce villifera (Scheele) Small Fl. SE. U. S. 7I2, I333. I903.

The previously known range of this striking species, Texas to Orizaba, Mexico, is extended farther south by a number of specimens collected by Dr. Gaumer at Buena Vista Xbac and Chichankanab, Yucatan, IIO3, II $86,2229$.

\section{Chamaesyce yucatanensis $\mathrm{sp}$. nov.}

Rosulate-prostrate from a slender annual root; stem and branches filiform, pilose. Inflorescence solitary in the axils of the upper leaves. Leaves inaequilateral, 5-7 $\times 2-4 \mathrm{~mm}$., oblong-lanceolate, strongly oblique at the base, acute, the margin distantly serrate; petioles about Imm. long; stipules represented below by a somewhat denser mass of the stem hairs, above by a scale-like, waxy gland at the base of the petiole. Involucres tubular-campanulate, sessile or apparently so, pilose without glabrous within; lobes represented by clusters of hairs except where flanking the deep, round-based sulcus where they are triangular, acute; glands bright-green, scutelliform, appendages white, unequal, about twice the size of the glands, ovate, unequally crenate-margined; bracteoles none; male pedicels $3-5$; female pedicel about the length of the ovary; styles 3 , bifurcate at the upper third; ovary pilose. Capsule pilose, ovoid, deeply sulcate; seed pinkish-ashen, $.8 \times .5 \mathrm{~mm}$., sharply angled, the facets marked by irregular and broken transverse ridges.

Near Ch. cozumelensis.

Progreso, Yucatan, on the rocky plain south of the lagoon, Millspaugh I696, March 5, I899. Type in herb Field Mus. cat. no. 61,696. Erroneously referred to Ch. Karwinskyi (Boiss.) (E. Karwinskyi Boiss.) in Field Mus. Bot. 2:65.

The other North American species of this genus not previously transferred to it are:

Chamaesyce acuta (Engelm)

Euphorbia acuta Engelm. Mex. Bound. 189. 
408 Field Museum of Natural History - Botany, Vol. II

Chamaesyce albescens (Urban)

Euphorbia portoricensis albescens Urban Symb. Antill. I:339.

Chamaesyce ammatotricha (Boiss.)

Euphorbia ammatotricha Boiss. Cent. Euph. Io.

Chamaesyce Andromedae (Millsp.)

Euphorbia Andromedae Millsp. Field Mus. Bot. 2:63.

Chamaesyce anychioides (Boiss.)

Euphorbia anychioides Boiss. Cent. Euph. I2.

Chamaesyce arenicola (Parish)

Euphorbia arenicola Parish Erythea 7:93.

Euphorbia cuspidata Engelm. in herb. non Bertol.

Chamaesyce astyla (Engelm.)

Euphorbia astyla Engelm. Boiss. DC. Prodr. I5:40.

Chamaesyce Balbisii (Boiss.)

Euphorbia Balbisii Boiss. Cent. Euph. I I.

Chamaesyce bartolomaei (Greene)

Euphorbia bartolomaei Greene Pittonia I:290.

Chamaesyce Brandegeei (Millsp.)

Euphorbia Brandegeei Millsp. Proc. Calif. Acad. ser. 2, 2:226.

Chamaesyce bryophylla (Donn-Sm.)

Euphorbia bryophylla Donnell-Smith Bot. Gaz. 56:62.

Chamaesyce capitellata (Engelm.)

Euphorbia capitellata Engelm. Mex. Bound. 2:I88.

Chamaesyce carmenensis (Rose)

Euphorbia carmenensis Rose Contr. U. S. Nat. Herb. I:I33.

Chamaesyce centunculoides (Kth.)

Euphorbia centunculoides HBK. Nov. Gen. 2:4I.

Chamaesyce chalicophila (Wetherb.)

Euphorbia chalicophila Wetherb. Proc. Am. Acad. 45:426.

Chamaesyce chamaecaula (Wetherb.)

Euphorbia chamaecaula Wetherb. idem.

Chamaesyce conjuncta (Millsp.)

Euphorbia conjuncta Millsp. Proc. Calif. Acad. ser. 2, 2:227.

Chamaesyce consanguinea (Engelm.)

Euphorbia consanguinea Engelm. Mex. Bound. 187.

Chamaesyce crassinodis (Urban)

Euphorbia crassinodis Urban Symb. Antill. I:340.

Chamaesyce cumbrae (Boiss.)

Euphorbia cumbrae Boiss. Cent. Euph. r6r. 
Chamaesyce Deppeana (Boiss.)

Euphorbia Deppeana Boiss. Cent. Euph. 6.

Euphorbia Anthonyi Brandegee Erythea 7:7.

Euphorbia clarionensis Brandegee idem.

Although Deppe remarks "in California" he must have collected the plant on some one of the out islands near the California coast. The species has not been found on the mainland.

Chamaesyce dorsiventralis (Urban)

Euphorbia dorsiventralis Urban Symb. Antiill. 5:391.

Chamaesyce floribunda (Engelm.)

Euphorbia floribunda Engelm. Boiss. DC. Prodr. 15:39.

Chamaesyce florida (Engelm.)

Euphorbia florida Engelm. Mex. Bound. 189.

Chamaesyce fruticosa (Engelm.)

Euphorbia fruticosa Engelm. Boiss. DC. Prodr. 15:38.

Chamaesyce Garkeana (Boiss.)

Euphorbia Garkeana Boiss. DC. Prodr. 15:38.

Chamaesyce glyptosperma (Engelm.) Small Fl. SE. U. S. 7 I $2 ;$ I333.

Euphorbia Greenei Millsp. Pittonia 2:88.

Chamaesyce aequata Lun. Am. Midl. Nat. I:204.

Chamaesyce aequata claudicans Lun. ibid 205.

Chamaesyce glyptosperma integrata Lun. ibid 3:142.

Chamaesyce gracillima (Wats.)

Euphorbia gracillima Wats. Proc. Am. Acad. 21:438.

Chamaesyce grisea (Engelm.)

Euphorbia grisea Engelm. Boiss. DC. Prodr. 15:4I.

Chamaesyce gymnadenia (Urban)

Euphorbia gymnadenia Urban Symb. Antill. 5:390.

Chamaesyce Heraldiana nom. nov.

Euphorbia glaberrima Klotz. Seem. Bot. Herald. 276, non Koch.

Chamaesyce hirtula (Engelm.)

Euphorbia hirtula Engelm. Wats. Bot. Calif. 2:74.

Chamaesyce incerta (Brandegee)

Euphorbia incerta Brandeg. Proc. Calif. Acad. ser. 2, 3:171.

Chamaesyce interaxillaris (Fern.)

Euphorbia interaxillaris Fernald Proc. Am. Acad. 36:495.

Chamaesyce intermixta (Wats.)

Euphorbia intermixta Watson Proc. Am. Acad. 24:74. 
4 io Field Museum of Natural History - Botany, Vol. II

Chamaesyce involuta (Millsp.)

Euphorbia involuta Millsp. Proc. Calif. Acad. ser. 2, 2:227 et 3:I 70.

Chamaesyce Jonesii (Millsp.)

Euphorbia Jonesii Millsp. Pittonia 2:89.

Chamaesyce leucantha (Kl. \& Gke.)

Anisophyllum leucanthum K1. \& Gke. Tricocc. 27.

Chamaesyce leucophylla (Bth.)

Euphorbia leucophylla Bentham Bot. Sulph. 50.

Euphorbia velutina Greene Bull. Calif. Acad. ser. 2, 5:57.

Chamaesyce liliputiana (Wright)

Euphorbia liliputiana Wright Urban Symb. Antill. 5:390.

Euphorbia minutula Boiss. DC. Prodr. 15:1263.

Chamaesyce linearis (Retz.)

Euphorbia linearis Retz. Obs. 3:32.

Euphorbia articulata Aubl. P1. Gui. r:480.

Chamaesyce lineata (Wats.)

Euphorbia lineata Wats. Proc. Am. Acad. 21:454.

Chamaesyce longeramosa (Wats.)

Euphorbia longeramosa Watson Proc. Am. Acad. 25:161.

Chamaesyce luisensis nom. nov.

Euphorbia tomentella Engelm. Boiss. DC. Prodr. 15:32, non Zipp. ex Span. in Linnaea $15: 350$.

Chamaesyce magdalenae (Benth.)

Euphorbia magdalenae Bentham Bot. Sulph. 50.

Chamaesyce melanadenia (Torrey)

Euphorbia melanadenia Torr. Pacif. R. R. Exped. 4:135.

Euphorbia polycarpa vestita Wats. Bot. Calif. 2:73.

Chamaesyce Mendezii (Boiss.)

Euphorbia Mendezii Boiss. Cent. Euph. I5.

Chamaesyce multinodis (Urban)

Euphorbia multinodis Urban Symb. Antill. I:34I.

Chamaesyce myrtillifolia (L.)

Euphorbia nuyrtillifolia Linn. Syst. ed. X:I048.

Chamaesyce occidentalis (Drew)

Euphorbia occidentalis Drew Bull. Torr. Club 16:I 52.

Chamaesyce ocellata (Dur. \& Hilg.)

Euphorbia ocellata Dur. \& Hilg. Pacif. R. R. Rep. V:5:I 5.

Chamaesyce paucipila (Urban)

Euphorbia paucipila Urban Symb. Antill. 5:389. 
Chamaesyce petrina (Wats.)

Euphorbia petrina Watson Proc. Am. Acad. 24:75.

Chamaesyce picachensis (Brandeg.)

Euphorbia picachensis Brandegee Univ. Calif. Bot. 6:185.

Chamaesyce pileoides (Millsp.)

Euphorbia pileoides Millsp. Field Mus. Bot. 2:62.

Chamaesyce podadenia (Boiss.)

Euphorbia podadenia Boiss. DC. Prodr. 15:40.

Chamaesyce polycarpa (Benth.)

Euphrobia polycarpa Bentham Bot. Sulph. 50.

Chamaesyce Pondii (Millsp.)

Euphorbia Pondii Millsp. Contrib. U. S. Nat1. Herb. I:I2.

Chamaesyce portulana (Wats.)

Euphorbia portulana Watson Proc. Am. Acad. 24:75.

Chamaesyce pseudoserpyllifolia (Millsp.)

Euphorbia pseudoserpyllifolia Millsp. Pittonia 2:87.

Chamaesyce puberula (Fern.)

Euphorbia puberula Fernald Proc. Am. Acad. ser. 2, 36:494.

Chamaesyce purissimana (Millsp.)

Euphorbia purissimana Millsp. Proc. Calif. Acad. ser. 2, 2:225.

Chamaesyce pycnanthema (Engelm.)

Euphorbia pycnanthema Engelm. Mex. Bound. 2:188.

Chamaesyce radicans (Moric.)

Euphorbia radicans Moric. in Berland. Pl. Exsic.

Euphorbia serpens radicans Engelm. Boiss. DC. Prodr. 15:30.

Chamaesyce radioloides (Boiss.)

Euphorbia radioloides Boiss. DC. Prodr. 15:45.

Chamaesyce ramosa (Seaton)

Euphorbia ramosa Seaton Proc. Am. Acad. ser. 2, 28:I 2 I.

Chamaesyce Rattanii (Wats.)

Euphorbia Rattanii Watson Proc. Am. Acad. 20:272.

Chamaesyce rubrosperma (Lotsy)

Euphorbia rubrosperma Lotsy Bot. Gaz. 20:349.

Chamaesyce Rusbyi (Greene)

Euphorbia Rusbyi Greene Bull. Calif. Acad. 57.

Chamaesyce scopulorum (Brandeg.)

Euphorbia scopulorum Brandegee Univ. Calif. Bot. 4:184.

Chamaesyce Seleri (Donn-Sm.)

Euphorbia Seleri Donn-Smith Bot. Gaz. 27:44I. 


\section{I 2 Field Museum of Natural History - Botany, Vol. II}

Chamaesyce thymifolia (Linn.)

Euphorbia thymifolia Linn. Sp. P1. 454.

Chamaesyce tomentulosa (Wats.)

Euphorbia tomentulosa Watson Proc. Am. Acad. 22:476.

Chamaesyce tonsita nom. nov.

Euphorbia polycarpa hirtella Boiss. DC. Prodr. I5:44, non E. hirtella

Boiss. Cent. Euph. 7.

Chamaesyce polycarpa hirtella Millsp. Parish Cat. P1. Salton Sink 6.

Chamaesyce Torralbasii (Urban)

Euphorbia Torralbasii Urban Symb. Antill. I:340.

Chamaesyce trachysperma (Engelm.)

Euphorbia trachysperma Engelm. Mex. Bound. 2:189.

Chamaesyce Turpini (Boiss.)

Euphorbia Turpini Boiss. Cent. Euph. I2.

Chamaesyce umbellulata (Engelm.)

Euphorbia umbellulata Engelm. Boiss. DC. Prodr. I 5:40.

Chamaesyce velleriflora (Kl. \& Gke.)

Anisophyllum velleriflorum K1. \& Gke. Tricocc. 28.

Euphorbia velleriflora Boiss. DC. Prodr. I5:40.

Chamaesyce velligera (Schaur.)

Euphorbia velligera Schaur. in Linnea 20:728.

Chamaesyce vestita (Boiss.)

Euphorbia vestita Boiss. Cent. Euph. 7 .

Chamaesyce villosior (Greenm.)

Euphorbia villosior Greenman Proc. Am. Acad. ser. 2, 32:297.

Chamaesyce Urbanii nom. nov.

Euphorbia villosula Urban Symb. Antill. I:340 (I899),

non Pax Bot. Yahrb. I9:I 8 (I894).

Chamaesyce Watsoni (Millsp.)

Euphorbia Watsoni Millsp. Zoe I:347.

Eumecanthus K1. \& Gke. Monat. Akad. Berl. 1859:248.

Leaves aequilateral, usually sparse below, the upper opposite; inflorescence solitary or cymose; bracteoles obsolete; seeds ovoid-globose, deeply and regularly honeycomb-pitted each pit with a secondary pit or papule in its fundus.

Type species: Euphorbia ariensis HBK.

LEPTOPUS Kl. \& Gke. ibid 249, non Decne.

Adenopetalum K1. \& Gke. ibid 250, non Turcz. 
Eumecanthus Armourii.

Euphorbia Armourii Millsp. Field Mus. Bot. I:28. I895.

The range of this species is Cozumel Island and the main land of Yucatan from Merida to Izamal, San Anselmo, Chichan Itza and Buena Vista Xbac. Schott 76; Millspaugh 108, I569, I570; Gaumer 975, I821; Seler 3927, 4012; Goldman 536.

Eumecanthus astroites (Fisch. \& Mey.).

Euphorbia astroites F. \& Mey. Anim. Ind. Sem. Petrop. 1845, Ann. Soc. Nat. ser. 3, 5:37I.

Euphorbia astroites heterappendiculata Millsp. Field Mus. Bot. I:28. I895.

The range of this species is: Mexico at Tampocoala, Karwinsky; Izamal, Millspaugh 64, Gaumer 521; and Guatemala, Kellerman 8038.

Eumecanthus gramineus (Jacq.) comb. nov.

Euphorbia graminea Jacq. Stirp. Amer. I5 r. ${ }^{7} 763$.

This species has a broad distribution from Chihuahua, Mexico, southward to U. S. Colombia and Ecuador. In Yucatan it has been returned from Calotmul, Izamal, Tekax, Temax, Yaxcab and Chichankanab.

\section{Eumecanthus xbacensis.}

Euphorbia xbacensis Millsp. Field Mus. Bot. r:374. r898.

Only known from the type station, Buena Vista Xbac, Yucatan, Gaumer I I08, "rare."

The other North American species are:

Eumecanthus ariensis (Kth.) Kl. \& Gke. Tricocc. 42. I859.

Euphorbia ariensis Kunth. HBK. Nov. Gen. 2:46. I8I 7.

Eumecanthus Benthamianus K1. \& Gke. idem.

Eumecanthus adiantoides (Lam.)

Euphorbia adiantoides Lam. Encyc. 2:422.

Leptopus Hartwegii K1. \& Gke. Tricocc. 46.

\section{Eumecanthus Barnesii}

Adenopetalum Barnesii Millsp. Field Mus. Bot. 2:377.

Eumecanthus bifurcatus (Engelm.)

Euphorbia bifurcata Engelm. Mex. Bound. 2:Igo.

Eumecanthus boerhaavifolius (Boiss.)

Euphorbia boerhaavifolia Boiss. DC. Prodr. I 5:54.

Eumecanthus bracteatus (K1. \& Gke.)

Adenopetalum bracteatum Kl. \& Gke. Tricocc. 48.

Euphorbia scabrella Boiss. DC. Prodr. 15:55. 
4I4 Field Museum of Natural History - Botany, Vol. II

Eumecanthus delicatulus (Boiss.)

Euphorbia delicatula Boiss. Cent. Euph. I9.

Eumecanthus dioscoreoides (Boiss.)

Euphorbia dioscoreoides Boiss. Cent. Euph. 22.

Eumecanthus discolor (Boiss.)

Euphorbia discolor Boiss. DC. Prodr. I5:54.

Eumecanthus Francoanus (Boiss.)

Euphorbia Francoana Boiss. Cent. Euph. 22.

Eumecanthus Hoffmanni (Kl. \& Gke.)

Adenopetalum Hofmanni K1. \& Gke. Tricocc. 47.

Eumecanthus digitatus (Wats.)

Euphorbia digitata Watson Proc. Am. Acad. 26:146.

Eumecanthus calcicolus (Fern.)

Euphorbia calcicola Fernald Proc. Am. Acad. ser. 2, 36:496.

Eumecanthus chiapensis (Brandeg.)

Euphorbia chiapensis Brandegee Univ. Calif. Bot. 6:54.

Euphorbia tetradenia Brandegee exsic. distrib.

Eumecanthus cofradianus (Brandeg.)

Euphorbia cofradiana Brandegee Zoe 5:207.

Eumecanthus colimae (Rose)

Euphorbia colimae Rose Contrib. U. S. Nat1. Herb. I:356.

Eumecanthus guadalajaranus (Wats.)

Euphorbia guadalajarana Watson Proc. Am. Acad. 22 :449.

Eumecanthus humayensis (Brandeg.)

Euphorbia humayensis Brandegee Zoe 5:208.

Eumecanthus latericolor (Brandeg.)

Euphorbia latericolor Brandegee Univ. Calif. Bot. 4:377.

Eumecanthus mexicanus (Kl. \& Gke.)

Adenopetalum mexicanus Kl. \& Gke. Tricocc. 50.

Eumecanthus microappendiculatus (Lotsy)

Euphorbia microappendiculatum Lotsy Bot. Gaz. 20:349.

Eumecanthus monanthus (Wr.)

Euphorbia monantha Wright P1. Cub. Griseb. I866.

Eumecanthus multisetus (Bth.)

Euphorbia multiseta Benth. Pl. Hartw. 51.

Eumecanthus muscicolus (Fern.)

Euphorbia muscicola Fernald Proc. Am. Acad. 36:495.

Eumecanthus ocymoideus (L.)

Euphorbia ocymoidea Linn. Sp. Pl. 453. 
Eumecanthus pedunculosus (A. Rich.)

Euphorbia pedunculosa A. Rich. Hist. Cuba I I:I98.

Eumecanthus physalifolius (Boiss.)

Euphorbia physalifolia Boiss. Cent. Euph. 19.

Eumecanthus pubescens (Kl. \& Gke.)

Adenopetalum pubescens K1. \& Gke. Tricocc. 49.

Adenopetalum irasuense K1. \& Gke. ibid. 50.

Eumecanthus segoviensis (Boiss.)

Euphorbia segoviensis Boiss. DC. Prodr. 15:58.

Eumecanthus sinaloensis (Brandg.)

Euphorbia sinaloensis Brandegee Zoe. 5:208.

Eumecanthus subpeltatus (Wats.)

Euphorbia subpeltata Watson Proc. Am. Acad. 25:I46.

Eumecanthus subreniforme (Wats.)

Euphorbia subreniforme Watson Proc. Am. Acad. 2 I:438.

Eumecanthus subsinuatus (Boiss.)

Eupinorbia graminea subsinuata Boiss. DC. Prodr. 15:54.

Eumecanthus tenerus (Wats.)

Euphorbia tenera Watson Proc. Am. Acad. 18:150.

Eumecanthus triphyllus K1. \& Gke. Tricocc. 43.

Euphorbia triphylla Willd. Herb. 9316.

Euphorbia scabrella Boiss. DC. Prodr. I5:55.

Eumecanthus umbrosus (Bertero)

Euphorbia umbrosa Bertero Spr. Syst. 3:79I.

Eumecanthus violaceus (Greenm.)

Euphorbia violacea Greenman Proc. Am. Acad. ser. 2, 33:480.

Eumecanthus xalapensis (Kth.)

Euphorbia xalapensis HBK. Nov. Gen. 2:48

Eumecanthus zierioides (Boiss.)

Euphorbia zierioides Boiss. DC. Prodr. 15:58.

AkLema Rafin. Fl. Tell. 4:II4. I836-I838.

Branches articulate; leaves aequilateral, ternate or verticillate; stipules glandular; inflorescence in axillary and terminal cymes, corymbs or panicles; bracteoles numerous, plumose; seeds ovoid-globose, shallowly open and regular pitted with no secondary pits.

Type species: Euphorbia nudiflora Jacq.

Euphorbia Linn. Sp. P1. 453, in part. 1753.

Alectoroctonum Schlecht. in Linn. I9:252. 1847.

Peccana Rafin. Sylv. Tell. i 4 . $\quad$ i838. 
4 i6 Field Museum of Natural History - Botany, Vol. II

\section{Aklema Gaumeri}

Euphorbia Gaumeri Millsp. Field Mus. Bot. I:372.

The only known range of this species is Yucatan, at San Anselmo and Buena Vista Xbac, Gaumer 1039, I073, I443.

\section{Aklema Mayana}

Euphorbia Mayana Millsp. Field Mus. Bot. I:304.

Only known from Yucatan, at Izamal and Buena Vista Xbac Gaumer 302, 1039 .

The other North American species are:

Aklema adinophylla (Donn-Sm.)

Euphorbia adinophylla Donn-Smith Bot. Gaz. 47:26r.

Aklema apocynoides (Kl.)

Euphorbia apocynoides Klotzsch Seem. Bot. Herald 99.

Aklema colletioides (Bth.)

Euphorbia colletioides Benth. Sulph. Voy. I63.

\section{Aklema comonduana}

Euphorbia comonduana Millsp. Proc. Calif. Acad. ser. 2, 2:229. Aklema cotinifolia (L.)

Euphorbia cotinifolia Linn. Sp. P1. 453.

Aklema elliptica (Kl. \& Gke.)

Adenopetalum ellipticum K1. \& Gke. Tricocc. 50.

Euphorbia saccharata Boiss. Cent. Euph. I8.

Aklema Friderichthalii (Boiss.)

Euphorbia Friderichthalii Boiss. DC. Prodr. 15:6r.

Aklema ligustrina (Boiss.)

Euphorbia ligustrina Boiss. Cent. Euph. 22.

Aklema Nelsonii

Euphorbia Nelsonii Millsp. Bot. Gaz. 26:268.

Aklema nudiflora (Jacq.) Rafin. Fl. Tell. 4:I 4.

Euphorbia nudiflora Jacq. Coll. 3:180.

Aklema oaxacana (R. \& G.)

Euphorbia oaxacana Robn. \& Greenm. Proc. Am. Acad. 32:37.

Aklema ovata (Schlecht.)

Alectoroctonum ovatum Schlecht. in Linn. I9:252.

Euphorbia Schlechtendahlii Boiss. Cent. Euph. I8.

Aklema peganoides (Boiss.)

Euphorbia peganoides Boiss. Cent. Euph. 22.

Aklema petiolare (Sims) Millsp. Ann. Mo. Bot. Gard. 2:43. I915. Euphorbia petiolaris Sims. Bot. Mag. t. 883 . 
Aklema plicata (Wats.)

Euphorbia plicata Watson Proc. Am. Acad. 2 I:438.

Aklema Scotana (Schlecht.)

Alectoroctonum Scotanum Schlecht. in Linn. I9:252.

Euphorbia Scotana Boiss. DC. Prodr. I5:60.

Aklema tricolor (Greenm.)

Euphorbia tricolor Greenm. Proc. Am. Acad. 33:479.

Aklema Xanti (Engelm.)

Euphorbia Xanti Engelm. Boiss. DC. Prodr. 15:62.

Euphorbia gymnoclada Engelm. Proc. Am. Acad. 5:I 7I, non Boiss.

Aklema yavalquahuitl (Boiss.)

Alectoroctonum yavalquahuitl Schlecht. in Linn. I9:252.

Acalypha flagellata sp. nov.

A dioecious shrub, 2-5 meters high, glabrous or nearly so, with terete, pithy branches, large leaves $\left(7.5 \times 12 \mathrm{~cm}\right.$.) and very long spikes $\left(\mathrm{I}_{5}-35\right.$ cm.).

Stipules leafy, narrowly ovate-lanceolate, serrate, apiculate, $\mathrm{I}-2 \mathrm{~cm}$. long; petioles 6-7 cm. long; blade palmately 5 -veined, ovate, acuminate, thin, minutely wrinkle-pitted in drying, crenate-dentate, each tooth mucronate. Infloresence in long, simple, lash-like, axillary spikes often in pairs on the male shrubs though generally single, rarely one monoecious spike appears, this always on the female shrub. Female flowers single, on a long, filamentous pedicel $(8-\mathrm{I} 2 \mathrm{~mm}$.); pedicel bibracteate, pilose; calyx 4-parted the lobes linear-lanceolate, ciliate, acute; ovary hairy and stalked-tuberculate; stylar column glanduliferous, turbinate, the upper portion cut into three lobes from each of which issues a fascicle of six long, filiform, transparent stigmas. Male flowers densely fasciculate on slender, cylindrical spikes, very short pedicellate; sepals 4 , scaphoid, minute, caducous; stamens 8, filaments ligulate, ciliate. Capsule $5 \mathrm{~mm}$. diam. deeply tricoccous the cocci globular, strongly marked by the line of dehiscence, scurfy and stalked-glanduliferous-tuberculate beneath; seed red-brown, globose, apiculate, I.2 $\mathrm{mm}$. diameter, minutely papillate-carunculate, the surface minutely puncticulate.

Type station Buena Vista Xbac, Yucatan, Gaumer, September I 896, IIO7 (Type in herb. Field Museum, cat. no. 438,224). Also gathered by Dr. Gaumer at Chichankanab $2178,2179$.

Acalypha simplicissima sp. nov.

An erect, simple, slender annual about $4.5 \mathrm{~cm}$. high. Stem terete, crispidulous-hairy. Petioles filiform about the length of the thin, ovate, 


\section{4r8 Field Museum of Natural History - Botany, Vol. II}

acute leaves; leaves $3 \times 1.5-4 \times 2.5 \mathrm{~cm}$., glabrous, palmately 5-nerved at the rounded base, margin slightly crenate-dentate. Inflorescence axillary, the female flowers on long $(3-6 \mathrm{~cm}$.), pedunculate, wiry, ro-r 8bracted, open spikes; the male on a short, rudimentary, capitulate spike separate in the same axils. Bracts of the female flowers 6-7 $\mathrm{mm}$. ovate, narrowed to the sessile base, acute, the margin few-serrate, ciliate. Female flowers usually 3 to the bract, sessile; calyx 3-parted, lobes narrowly lanceolate; styles 6, fibrillate, about four times the length of the ovary. Male flowers ro or more in a dense, pedunculate head; calyx of four linear lobes with transparent, chaff-like margins. Capsule strigose-hairy; seed ashen, smooth, ovoid-apiculate, $\mathrm{x} .2 \times$ I mm.

Yucatan, at Progreso in a shady coppice, Gaumer II82 (Type in herb. Field Museum, cat. no. 438,255), and at Chichankanab, Gaumer 2225.

Croton Gaumeri sp. nov.

Dioecious, fruticose, open stellate-pubescent. Leaves thin, lanceolate-acuminate, $3-4.5 \times .75^{-1} .50 \mathrm{~cm}$.; petioles one-third the length of the blade; male shrubs with leaf margins distantly glandulose, on some individuals these glands become long-stalked though by no means on all nor on all leaves on any one shrub. Leaves (male) papillate on both surfaces, the margin generally distantly glandulose and often stalkedglandular especially near the base, in young leaves both surfaces are openly stellate-pubescent; stipules triangular, seldom found at the older leaves; leaves on female shrubs scattered stellate-pubescent above, more closely and prominently so beneath. Inflorescence terminal, in long, slender spikes floriferous above, flowers pedicellate, bracts minute, the male spikes slightly pubescent the female decidedly so and with long, straight hairs mixed with the closely appressed stellate ones; male flowers: calyx stellate-hairy, 4-lobed, the lobes ovate, acute, rudimentary petals 4 , lanceolate, stamens about 20 , filaments glabrous; female flowers: pedicels half the length of the calyx, calyx stellate-pubescent, 5-lobed, lobes oblong-lanceolate, acute, margin glandular, rudimentary petals 5 , alternate with the calyx lobes, narrowly ligulate and tipped with a globular gland, appearing like stamens, styles 3 divided to the base, digitately 4 -lobed, lobes cylindrical, incurved at the tip. Ripe capsule about $5 \mathrm{~mm}$. diam., smooth as if varnished and bearing a few isolated stellate hairs; seed scaraboid, smooth and shining greenishbrown, $3.5 \times 2.8 \mathrm{~mm}$., caruncle strongly reniform, thin, waxen.

Yucatan, vicinity of Izamal. Unnumbered specimens collected by Dr. Gaumer in I894 (Type in herb. Field Museum cat. no. I 87,265). Two sheets of twigs of male shrubs appear in the series of Dr. Gaumer's 
Izamal collection of $\mathrm{r} 888$ deposited in Kew Herbarium; duplicates of these in herb. Field Museum bear its cat. nos. I8r,499 and I8I,500.

\section{Croton glandulosepalus sp. nov.}

A tall, white-barked shrub with long, terminal spikes and thin, ovate-lanceolate leaves clustered toward the tips of the branchlets leaving the branches bare. Leaves ovate-lanceolate, sparingly appressed-stellate, acute to acuminate, apiculate, $4-6 \times 2-3 \mathrm{~cm}$., 3-nerved at the base, both surfaces dotted with scattering, fine, slender, stellate hairs which nearly disappear when the leaves are fully mature; petioles slender, about one-third the length of the blade, puberulous; stipules aristate, about $5 \mathrm{~mm}$. long. Inflorescence monoecious, terminal in elongate, rather openly flowered spikes with large, ovate bracts bearing a number of long-stalked, ciliate glands on the margin. Male flowers short-pedicellate enclosed by the scaphoidally enveloping bracts, calyx 4-lobed, woolly with stellate hairs, lobes ovate, petals 4 , thin, ovate-scaphoid, glabrous or nearly so; stamens ro, filaments short (about the length of the anthers), glabrous, anthers large, innate. Female flowers: thick pedicelled and densely white-woolly below; calyx 4-lobed the lobes ovate-lanceolate, sarcous, exteriorly invested with a dense mass of red, stalked-globular glands and enclosing the pistil closely; petals small, flabelliform, margin stalk-glandular; ovary densely white-stellate woolly; styles 3, each 4-cleft to the base, the filamentous branches incurled at their tips. Capsule and seed unknown.

Yucatan, common on the open plain from Progreso to Merida, Gaumer II54 (Type in herb. Field Museum cat. no. 438,492). Also returned by Dr. Gaumer from Buena Vista Xbac, 1046 and from Chichankanab 2204.

\section{Croton malvavisciifolius sp. nov.}

Shrub with terete, ferrugineous branches, large bi-colorate, irregularly and doubly serrate-dentate leaves, and short monoecious terminal spikes. Stems rusty stellate-hairy the central ray of the stellae erect and acicular the base appressed to the bark; stipules ligulate; petioles somewhat less than one-third the length of the leaf blade. Leaves broadly ovate, 7-I4 $\times$ 5-10 $\mathrm{cm}$., acute to acuminate, palmately 7 -nerved, dark-brown, papillate and scattered stellate-hairy above, light-green and densely felted with stellate hairs beneath, each leaf bearing 2 funnel-shaped, long, stalked glands at the petiole, base strongly cordate, apex acute, margin irregularly doubly-serrate, dentate with a stalked, funnel-shaped gland in the sinus of each of the larger dentations. Inflorescence monoecious, terminal, short, female below male above, 
densely ferrugino-stellate pubescent. Female flower short-pedicellate, bicolorate (dark within light without) color and pubescence a counterpart of that of the leaves; calyx lobes 5 , spatulate, deeply 3-5-dentate toward the apex; ovary ovate, rusty-hairy, styles 3 each bifid to the base, the branches long and cylindrical, with flattened, incoiled apices. Male flower bicolorate as in the female, pedicellate; calyx 5 -lobed the lobes ovate, obtuse; petals 5 , obovate-spatulate, thin, red-brown; stamens 6 , filaments with a tuft of hairs at the base. Capsule densely stellatepubescent, enclosed in the persistent calyx; seed flatly scaraboid, $3 \times 2$ $\mathrm{mm}$., caruncle very thin, papyraceous.

Yucatan, at Yot Tzonot, Gaumer I3I9 (Type in herb. Field Museum cat. no. 438,278 ).

\section{Tragia (Eutragia) yucatanensis sp. nov.}

An erect, shrubby climber, I meter high. Stem terete, puberulent, branches virgate; stipules small, cordate-lanceolate, acute, closely appressed to the stem; petioles one-third to one-half the length of the leaf blade; leaves ovate-lanceolate, 3-6 2 $^{-4} \mathrm{~cm}$., 5-nerved, somewhat cordate at the base, acuminate, dentate, scattered-hairy on both surfaces. Inflorescence in few flowered, slender, short, simple racemes one-half to three $\mathrm{cm}$. long, inserted opposite the upper leaf petioles, the female solitary near the base of the raceme, the male few, terminal; bracts minute, sessile, lanceolate, acute, entire. Male flowers pedicelled; pedicel about equaling the width of the calyx, pilose, somewhat thickened below; calyx 3-parted, lobes ovate, acute, glabrous; stamens 3, filaments turgid-bulbous below; anthers ovate; rudimentary ovary wanting. Female flowers pedicelled; pedicel about one-half the width of the calyx; calyx segments 6, ovate, acute; ovary 3-celled, densely acicular-hairy; style turgid, trifurcate to the middle, the branches tightly recurved at the tips. Capsule densely invested with long, white, acicular hairs; seed globose, $2.3 \mathrm{~mm}$. diameter, yellow, mottled in irregular anastomosing splotches and stippled with minute dots of red-brown.

Yucatan, in woodlands at Chichankanab, Gaumer 2154 (Type in herb. Field Museum cat. no. 438,347); and at San Anselmo, I93I. This species and Tragia nepetaefolia are called $\mathrm{P} \circ \mathrm{p} \circ \mathrm{x}$ by the Mayas. 


\section{VEGETATION OF ALACRAN REEF}

The Alacran Reef forms the northeasternmost danger on the Campeche Bank, in the Gulf of Mexico, 75 miles N. by E. I/2 E. from Sisal Fort on the Yucatan mainland. The position of Perez Cay, its most southern islet, is latitude $22^{\circ} 33^{\prime} 36^{\prime \prime} \mathrm{N}$.; longitude $89^{\circ} 4 \mathrm{I}^{\prime} 45^{\prime \prime} \mathrm{W}$.

The general form of the reef is semilunar, convex to the NE., the straight western face trending NNW. I/2 W. I 4 miles, its greatest width being 8 miles. The weather, or NE. side, is composed of a compact mass of coral, dry in many places, over which, on all parts, the sea breaks with violence. The lee side, similar to all reefs of this character in these seas, is composed of detached coral heads, easily seen in the clear, deep water of the bank.

At the SE. extreme of the reef are three small, sandy cays rising not over $5 \mathrm{ft}$. high. These are known as Perez, Pajaros and Chica Cays. Pajaros and Chica lie $1 / 4$ mile apart 800 yds. from the SE. extremity of the reef, Perez lying about a mile westward from them. Between the eastward pair and Perez there is an excellent reef-harbor for small vessels; formerly 4 fathoms could be carried into this harbor but at the time of our visit it held, in a few places, but a precarious 2 fathoms.

NW. by N., 3 miles from Perez, is a small cay, about 8 feet high, which we named Allison Island, and about the same distance southward of the NW. end of the reef is another islet, about ro feet high, which we named Utowana Cay, though we were unable to land upon it on account of sea conditions at the time of our approach.

The Alacran Bank is everywhere so steep-to that there is no safe anchorage for larger vessels, not even on the lee side, the only clear landing place is on the inner side of Perez Cay at the mouth of the reefharbor mentioned.

The approach to this shoal, when working to windward, requires more than ordinary attention as the soundings near it do not give sufficient warning and the islets are invisible if the sea be high. As the Yacht Utowana (which the owner and master Mr. Allison V. Armour allowed to approach the reef on the voyage of which I had the honor of being his guest) arrived in the neighborhood, the captain slowed the vessel down and sent a lookout to the cross trees. Though we proved to be but about two miles from Allison Island yet nothing could be seen of the reef until a cloud of frigate-birds* arose indicating its presence.

As the reef lies directly within the sweep of the Gulf Stream in the youth of its course we judged that it might receive aquavectent seeds

*Fregata aquila (Linn.). 
422 Field Museum of Natural History - Botany, Vol. II

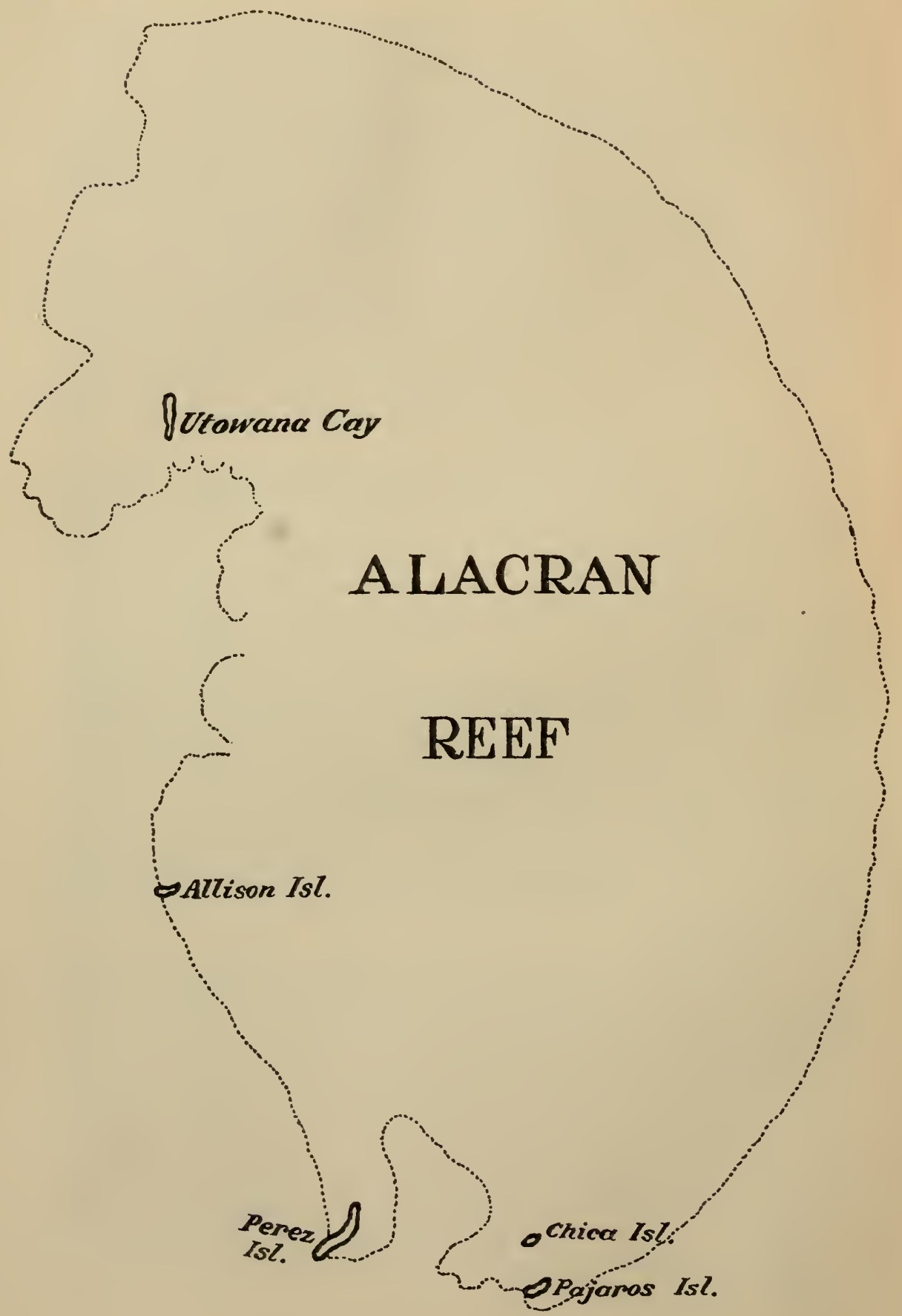





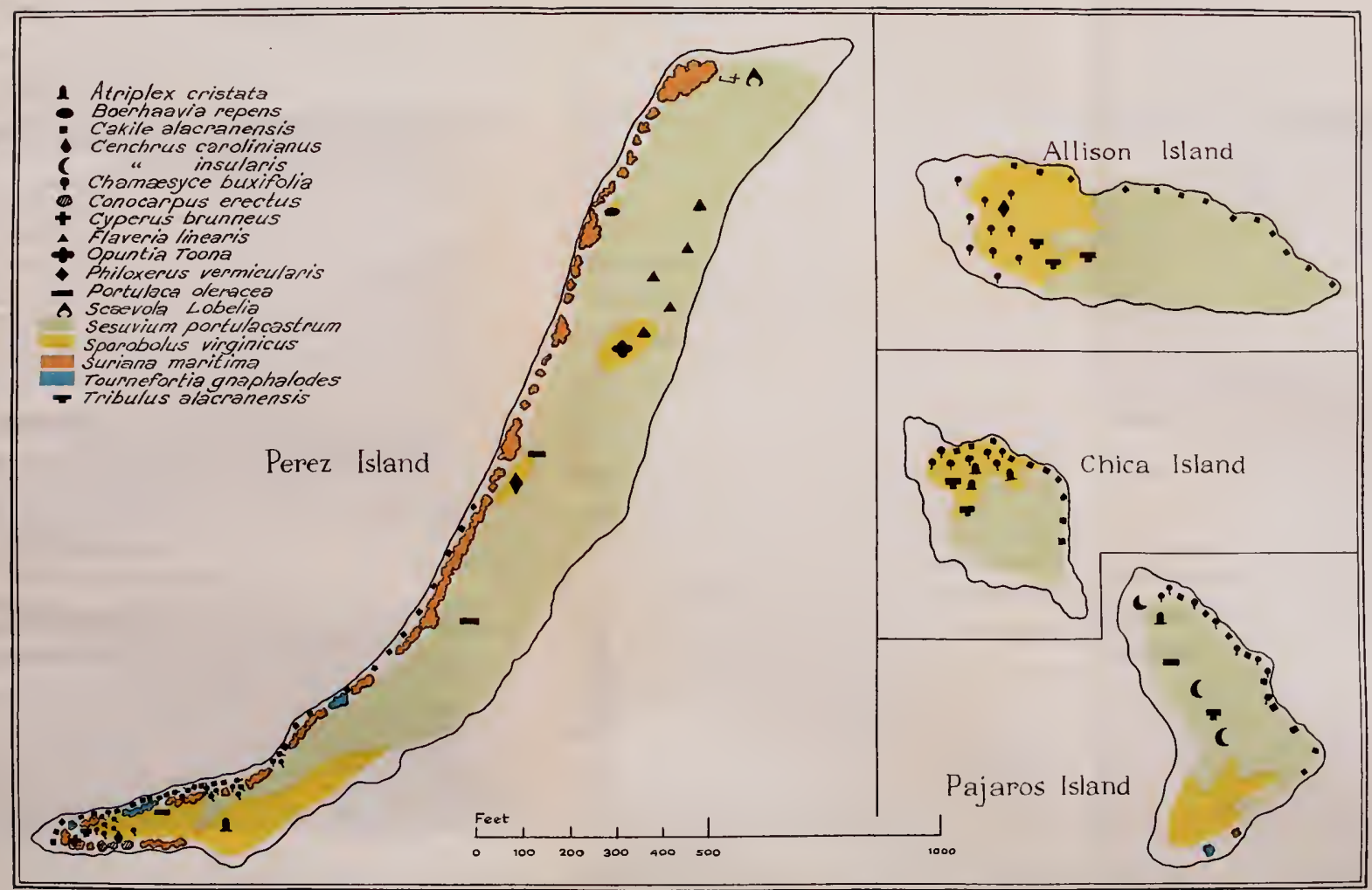


from the east coast of Yucatan, Cozumel Island, and the Caribbean mainland; and, on that account, desired to record its vegetation at this time (March, I899).

At the time of the charting of this reef by the British Admiralty, in I 842, Pajaros and Chica were "bare sand spits devoid of vegetation;" Perez, the largest cay, and Allison Island, had upon them "grass* and samphiret only." This record we are bound to accept as a careful and comprehensive one for the reason that on account of the dangerous character of the reef it was essential that it should be accurately described, even to its vegetation. Accumulated observations from 1842 to I 896 added "brushwood" to the southern cays and "bushes" to Allison Island, though no traces of bushes were to be found on the latter at the time of our visit; this indicates one of two facts: either that they had been removed, even to their roots, by fishermen for firewood, or by natural erosion. The age of the stunted trees termed "brushwood," as I have recorded further on under Suriana maritima, proves that "brushwood" was not on Perez in I 842 at the time of the Admiralty survey.

In searching out the vegetation of the cays a most careful survey was made. Each cay was minutely examined by our party over every square foot of its surface, I am certain that no species escaped our attention. A detailed indication of each of the eighteen species found is given in conjunction with the map.

The discovery, on these islets, of three species new to science, with the proof that they have evolved within the known and definite period of 57 years, is a fact impressive as it is important.

Perez Island, the largest cay, rises about 5 feet in height, its beach being more or less abrupt on the west and gradually sloping on the east or inner coast line. It is about $255^{\circ}$ feet in length and about 325 feet wide as its broadest point. Its surface is entirely coral sand except for a small cairn of weathered coral heads as indicated below.

I was informed that a few years previous to our visit a party of men was left on this cay, by the Mexican Government, with material for the purpose of constructing a beacon. Their supplies gave out and no more were sent to them. They were finally rescued by a fishing vessel and brought to the mainland in a dying condition. One died before the rescue and was buried at the north end of the cay. Several holes in the cay attested to the piteous attempts of these neglected men to secure

*Sporobolus virginicus.

†Sesuvium portulacastrum. 


\section{Field Museum of Natural History - Botany, Vol. II}

drinkable water. From the largest of these holes at the northern third of the islet a mound of weathered coral heads was heaped during its excavation. This forms the only prominence on the surface of the cay and is the habitat of a growth of cactus (Opuntia Toona) the seed of which was doubtless dropped here by some resting bird. In like manner a seed dropped from an arm of the little wooden cross marking the grave's head accounts for the only plant of Scaevola Lobelia. The "wooden, pyramidal framework 30 feet high" erected by this party at the south end of the islet, mentioned in the U. S. Coast Pilot for I 896, had long since disappeared as kindling for fishermen's cuddy fires.

The main extent of the surface is densely clothed, about 8 in. deep, with a level carpet of Sesuvium portulacastrum broken in only three places by small patches of Sporobolus virginicus associated, in one instance, with Portulaca oleracea; in another with Boerhaavia repens; and in a third with Flaveria linearis which has scattered in among the Sesuvium as mentioned under the consideration of this species. The whole of the south end is clothed with a nearly pure growth of the Sporobolus in which a few plants of Atriplex cristata have found a rooting.

The whole west, or weather, bank is lined with a fringe of Suriana which exists without the admixture of any other species for the northern two-thirds of its length; to the southward a few shrubs of Tournefortia intermingle and still further south six clumps of Conocarpus. Between the Suriana fringe, on the top of the bank, and the wave line of the narrow strand at the southern third, a scattering growth of the new Cakile, intermixed further southward with Chamaesyce buxifolia, completes the flora of the west shore. The interspersed species of the toe of the stocking-shaped islet, where boobies* evidently alight during fishing, were Cenchrus carolinianus, Portulaca oleracea and one individual each of Tribulus alacranensis and Cyperus brunneus.

For some reason neither the booby nor the frigate-bird, prevalent on the other cays, nest on this islet. The reason is not apparent to me unless mayhap the presence of the complete fringe of shrubbery on the west shore may prove, in some way, inimical to them.

Pajaros (Bird) Cay, the southeast islet of the shoal, is a low, pure sand microcosm, rounded to the center, with the eastern or weather shore line slightly banked and the western, or inner, beach sloping. It is about $650 \times 325$ feet in extent.

The distribution of plant species on its surface is in exact duplication of that on Perez; Cakile and Chamaesyce on the beach line of the weather shore and no plant life on the marge of the sloping strand.

* Sula cyanops (Sunderval). 


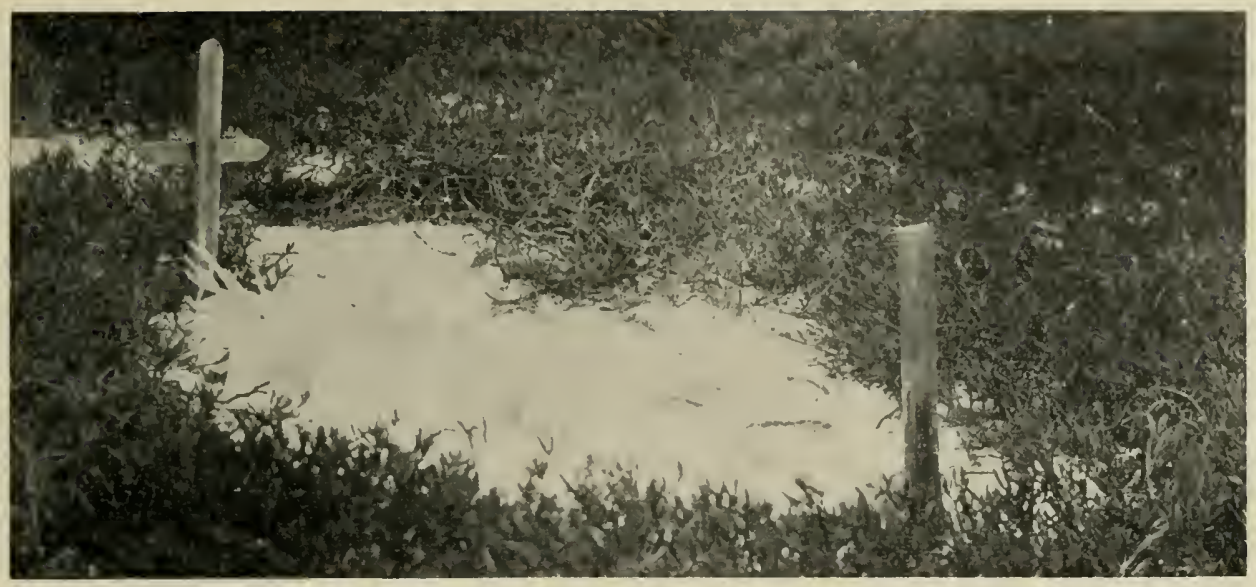

The grave on Perez IsLand.

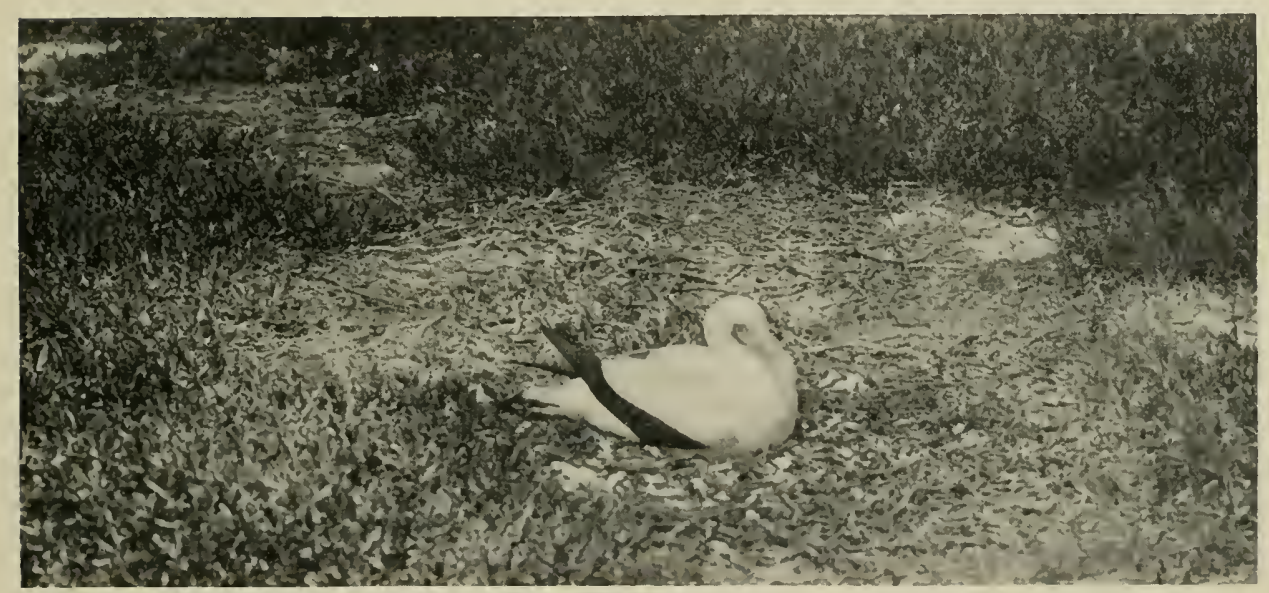

A BOOBY ON ITS NEST COMPOSED ENTIRELY OF THE STEMS OF TRIBULUS ALACRANENSIS.

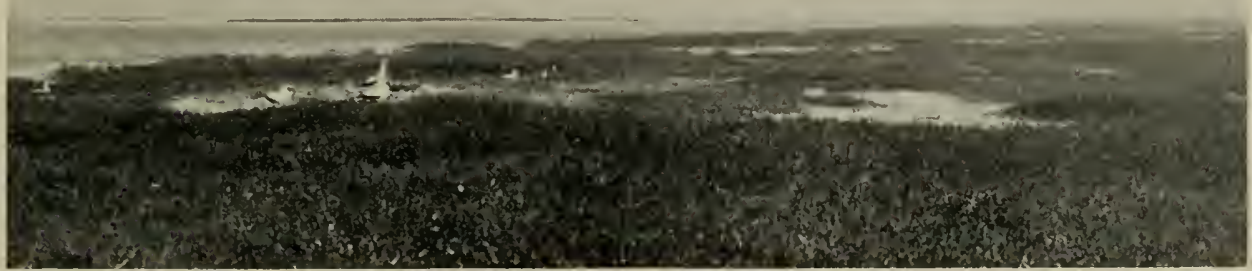

NORTHWEST END OF CHICA ISLAND CARPETED WITH SESUVIUM. LOOKING TOWARD PAJAROS ON THE HORIZON. 

Two shrubs only had secured a footing on this cay, one each of Conocarpus and Tournefortia. Scattered amongst the dense growth of Sesuvium was found a number of clumps of the new Cenchrus, forming its only habitat, and one clump each of the new Tribulus, Portulaca oleracea and Atriplex cristata. In the clear sand beyond the Sesuvium at its northernmost margin was another colony of the new Cenchrus.

ChICA (Little) Istand is another low, pure sand cay, fusiform in outline, $575 \times 325$ feet in extent.

On this islet the disposition of the Sesuvium and Sporobolus areas is reversed in comparison with the other two cays, while the scattering other species are interspersed with the Sporobolus colony instead of the Sesuvium. In this the clothing of the islet is quite similar to that of the foot of Perez and shows plainly that it forms a resting place for webfooted birds. Here the new Tribulus, Atriplex cristata and the Chamaesyce grow in profuse masses while the new Cakile maintains its characteristic habitat on the weather beach line.

Alurson Island, three miles from the other cays described, differs in several respects from the other three. First: it lies east and west-the others essentially north and south; second: its entire coast line is weather shore; i. e., abrupt and eroded above a narrow strand, except a small portion of its western extremity which is somewhat spit-like; third: it is considerably higher (about $8 \mathrm{ft}$.) and flat like a table-land. Its dimensions are about 1000 $\times 275$ feet.

Notwithstanding the fact that this islet is comparatively large it nevertheless gives place to but six plant species. Its "grass" and "samphire" colonies are pure, as on the other islets. The largest bird colonies of all are on this cay, in fact it is almost wholly a nidulation ground. Boobies appropriate the grass colony and frigate-birds the Sesuvium tract. It is on this cay that Tribulus alacranensis evolved, its presence on the other cays being a matter of transplantation through its burs having adhered to the feet of the booby. The species exists in dense colonies amongst the Sporobolus, and it is of its ligneous stems and branches that the boobies build their nests. The only colony of Philoxerus vermicularis on the cays is here in the Sporobolus, as well as a widely scattering growth of the Chamaesyce. Again the new Cakile lines itself along the weather strand but only on the weathermost side.

Utowana $C_{A y}$, which we sighted but were unable to attain, is the northernmost islet of the group. Through the glass its surface appeared very like that of Allison Island. As it lies north and south much further within the bank of the reef than any of the other cays its vegeta- 


\section{Field Museum of Natural History - Botany, Vol. II}

tion should prove particularly interesting, we were deeply disappointed that the risk was too great to allow of the yacht being brought near enough to grant us a landing.

\section{THE PLANT SPECIES}

I. Sesuvium portulacastrum (L.) Linn. Syst. ed. X, I058.

This is by far the prevalent species of the cays and here forms the most extensive and succulent masses I have ever seen. It clothes the easterly areas of all the islets with a dense carpet about eight inches thick. Only a few individuals were in flower at the time of our visit. As the stems of this plant are fragile; broken pieces long-lived; and as such pieces put forth strong and viril rootlets in water, I am inclined to place its dispersional character as bodily aquavectent. It is possible also that ripe fruits may be transported in like manner. Its characters are strongly against avevectence.

2. Sporobolus virginicus (L.) Kunth. Rev. Gram. I:67.

The second species in extent on the cays. This grass appears to spread only where some influence has checked the growth of the Sesuvium, as for instance the tramping about of the birds or the digging of water-holes and the constructive efforts of the men who built the beacon structure on the south end of Perez. This is so pronounced a fact that a glance at the distinguishing colors on the maps shows by the yellow, for Sporobolus, the location of the three water-holes and the area of the beacon-site; while the yellow on the maps of the other cays demarks perfectly the booby nesting areas - always, for some reason westerly on the islets. Note, by the same token, that the booby sections are always where the land slopes gently to spit-like extremities.* The booby never nests near the weather, or abrupt, shore.

From its habitat it is evident that Sporobolus is aquavectent; it has however only been found, on the Yucatan mainland, in protected situations near lagoons, whence, if its Alacran source is the mainland, its spikes must first have been blown to the sea before its real transportation began.

3. Suriana maritima L. Sp. Pl. 284.

A fringe of low shrubs of this species lines the top of the "bank" of the western or weather shore of Perez Island. The growth is pure in the northern two-thirds of the fringe, the southern third has an intermixture of a few clumps of Tournefortia and, further south, of Conocarpus.

* In order that the fledglings may get to the water and back to the nest? 

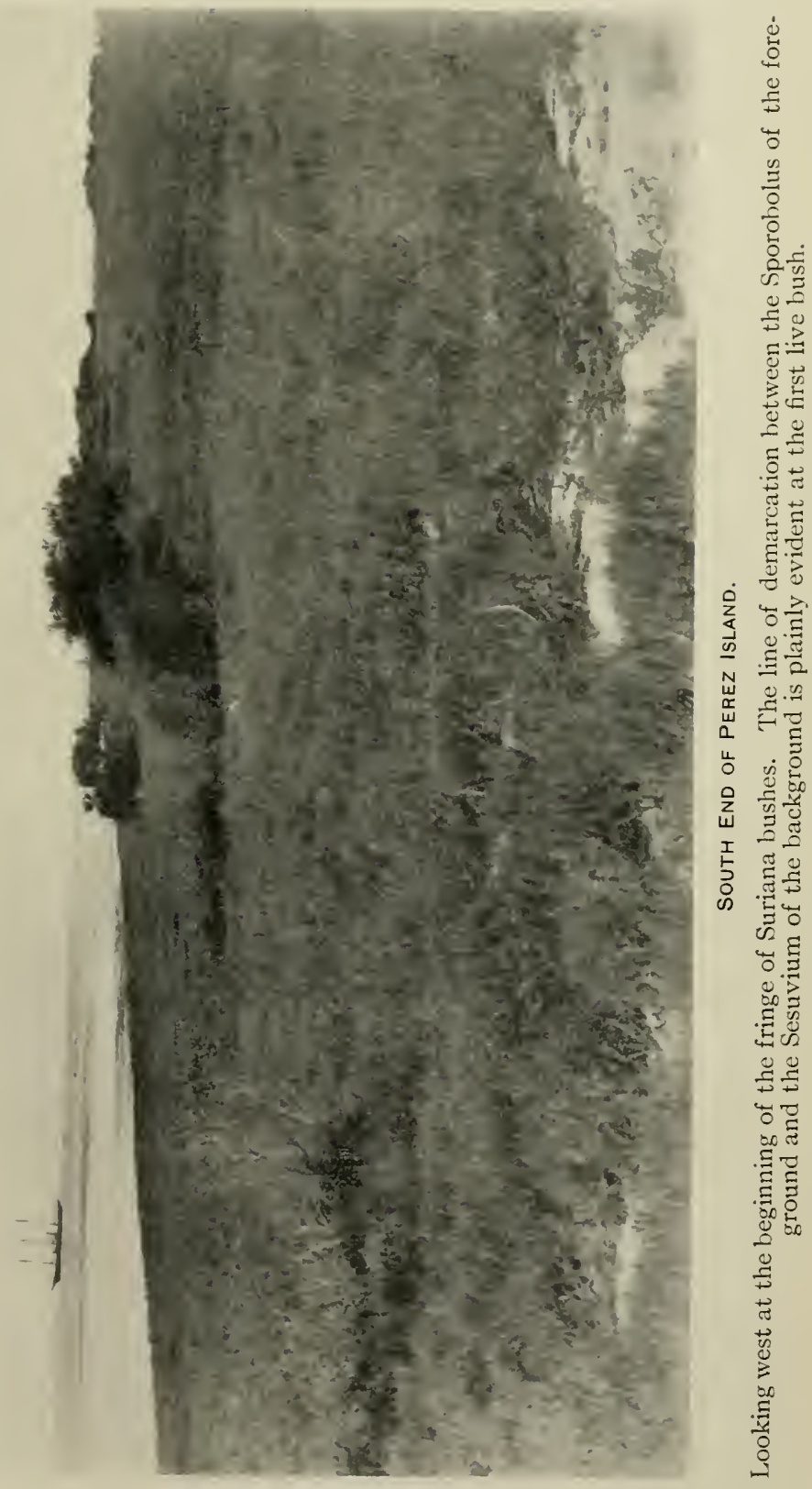

In order to check up the record of the Admiralty survey I cut down the two apparently oldest shrubs and made cross sections of the trunks.* In so far as the annual rings may determine they proved to be 25 and 30 years old respectively. Should the rings prove a faulty record the error would be in favor of a shorter period of growth, it is therefore certain that the shrubs did not exist there at the time of the survey, the notes of which state: "Grass and samphire only." One shrub, judged to be not over four years old, grew on the southern shore of Chica.

At the period of ripened fruits the fruiting twigs of this species become quite brittle and, as the plant frequents the immediate sea shore, these twigs are often broken off by heavy winds and blown into the sea. That they float away and are later washed ashore on the crests of waves was apparent in the "wash line" on the weather side of Perez where I found a large quantity of fruiting twigs newly come ashore. This leaves no doubt of the species being bodily aquavectent.

4. Cakile alacranensis Millsp. Field Mus. Bot. 2:130.

This new species of the shoal differs from all others known in its short silique bearing four channelled lines instead of 8 or more (angles in most species); in its upper joint being globose-apiculate and very turgid; in its infundibuliform lower joint which is but little more than a swelling of the pedicel, and in having its seeds dotted with brownish punctae. The leaves are yellowish-green, spatulate-lanceolate tapering to a partly clasping petiolar base, and entire or rarely slightly crenate or crenate-dentate at the apex.

This species grows upon the north strand of Allison Island; on the north weather strand of Chica and Pajaros; and on the southern half of

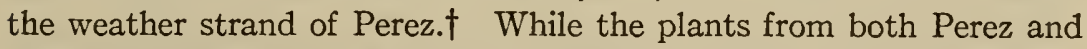
Pajaros are large fruit producers they have a sickly yellow appearance compared with the fresh, cool green of those of Chica. It is evident that the original habitat of this species is either Allison Island, or possibly unexplored Utowana Cay, as the distribution of the plants on the southern cays is quite apparently more recent.

It appears to me that this species is evolving for the purpose of more highly developed aquavectence. The upper, or floating joint, is certainly developing far more rapidly than the lower, or fixed, joint of the fruit: the upper at the expense of the lower.

Distributionally the upper joint of the fruit is aquavectent while the lower, being fixed upon the rhachis and on that account not adapted to

*These sections are mounted on the herbarium sheets of the species from this island in the Museum herbarium.

† The treatment of Cakile in Field Mus. Bot. 2:43 and 2:128-130 to the contrary notwithstanding. 


\section{Field Museum of Natural History - Botany, Vol. II}

dispersion, is evidently intended to perpetuate the species only in the habitat of the parent plant.

5. Chamaesyce buxifolia (Lam.) Small.

This common seaside Euphorbia is found, on the booby rest-strands only, on all the islets. It does not associate (except on Pajaros) with the aquavectent Cakile nor with other aquavectent species.

The seed coat of this species swells greatly and becomes mucilaginous when moist. This causes the seeds to adhere to the wet webs of swimming birds when resting along the shore. Thus the plants are widely distributed on the island strands of the Gulf of Mexico region.

6. Tribulus alacranensis Millsp. Field Mus. Bot. 2:54.

This new species differs from its probable parent, T. maximus of the mainland, in its long, heavy, tangled and jointed ligneous stems and branches; its mass growth; its larger flowers and smaller fruits; its 4-6-spined carpids; and in its leaves being densely woolly on both surfaces. The original habitat* of this species is apparently the Sporobolus colony of Allison Island, where the boobies construct their nests wholly of its stems and branches.

The dispersional character of the species is avevectent, through the sharp-spined fruits clinging to the webbed feet of swimming birds.

7. Tournefortia gnaphalodes R. Br. Prod. 496 .

This common sea shore shrub of the Antillean region has found a place of growth amongst the Suriana shrubs at the southern extension of the fringe on Perez. One shrub only was just appearing on the south shore of Pajaros. The specimens appear to differ in no respect from those of the mainland.

As with Suriana the fruiting branch tips of this species break off during high winds and are often blown into the sea, whence they are drifted to new places of growth. The species is, again like Suriana bodily aquavectent in dispersional characters. It is very seldom, if ever, found on the shores of bays or on shores guarded by partly dry reefs.

8. Conocarpus erectus Linn. Sp. P1. I47.

A few clumps of this shrub have established themselves amongst the Suriana bushes at the south end of the fringe on Perez Island, where the shore being free on the weather edge of the reef, there has accumulated quite a congregation of wave carried species.

Two of the largest trunks of this species showed, by the annual rings, a growth of 19 years.

*As Utowana Cay was not explored this statement is open to doubt. 
From its association on these cays I judge that the fruits are aquavectent. Its growth in no wise gives the appearance of bird transportation.

9. Flaveria linearis Lag. Gen. et Sp. Nov. 33 .

A few plants of this species were found near the coral cairn on Perez Island. A few more were found scattered in the Sesuvium carpet a short distance northwestward from the cairn. The species is fairly common on the coast of the mainland of Yucatan and on the island of Cozumel.

This dispersion of this species to the Alacran Reef must have been intestinally avevectent as the seeds have no coma and the position of the plants on Perez savors highly of resting birds.

10. Portulaca oleracea Linn. Sp. P1. 445.

A few individuals of this species were found scattered amongst the Sesuvium of Perez and Pajaros cays. It had not yet become implanted on the other cays visited.

In my studies of the intimate dispersion of species upon sand islets* I have found this species only upon inhabited islands and then generally in areas that were at the time, or had been, more or less cultivated for some purpose. The position of the plants on Perez and Pajaros in no way indicate homovectence, though on Perez this might have been possible. The dispersional indications in regard to this species are not sufficiently plain to me at this time to permit a distinct statement.

Ix. Cyperus brunneus Sw. Fl. Ind. Occ. I:Ir6.

One clump only of this species was found on the cays that on the southwest end of Perez Island just beyond the Sporobolus colony.

This species is quite a common element of the Antillean strand flora from the Bahamian Archipelago through to Vera Cruz, Mexico. It occurs in places all along the Yucatan coast and on the strands of the island of Mugeres.

The seeds are avevectent per pedis et intestinis.

r 2. Atriplex cristata H. \& B. ex Willd. Sp. P1. 4:959.

A group of a few individuals of this species was found in the Sporobolus colony on the south end of Perez and quite a large cluster in the same association on Chica. On Pajaros a small group was found in association with the Sesuvium. The species has also been returned from the Yucatan main coast at Progreso and Silam.

I judge that the fruits are avevectent, being transported on the webbed feet of the booby, and that the clump in the Sesuvium of Pajaros came originally from the older implantation on Chica.

\footnotetext{
* "Flora of the Sand Keys of Florida," Field Museum of Natural History, Botany, Vol. 2, no. 5 .
} 


\section{Field Museudi of Natural History - Botany, Vol. II}

I3. Cenchrus carolinianus Walt.

This species* was found only at one spot, that on the south shore of Perez Island near the clumps of Conocarpus at the edge of the Sporobolus colony at that point. The species has also been returned from Cozumel Island but not from the Yucatan mainland.

The species is avevectent through the spiny burs clinging to the webbed feet of the booby.

14. Cenchrus insularis Scribn. Field Mus. Bot. 2:26.

This new species is represented by a few plantst on Pajaros Island only. These were all in full ripe fruit. That this is the full representation of so strongly a differentiated species is open to doubt and will so

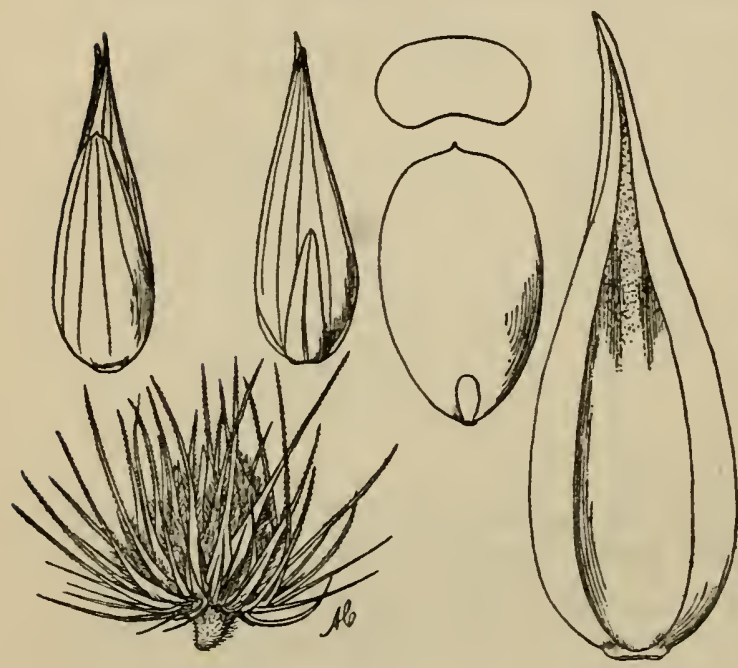

C. insularis.

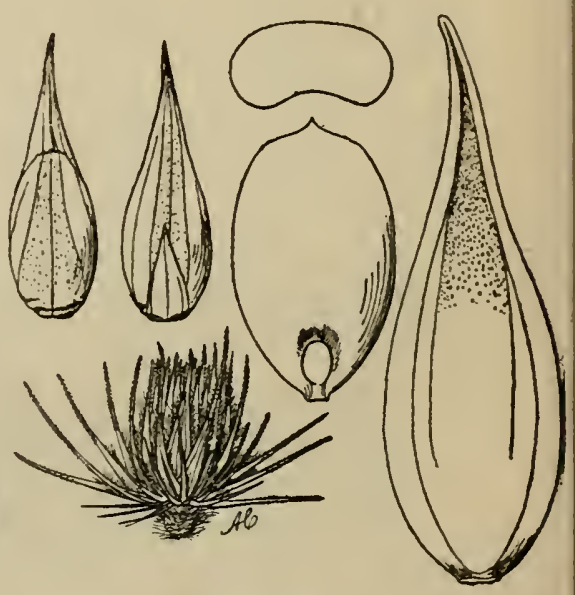

C. echinatus

remain until opportunity is had to visit Utowana Cay, where it would seem more than probable that a fuller growth may be found.

The characters clearly differentiate this species from $\mathrm{C}$. carolinianus as found on Perez Island; in many of its characters it is much nearer C. echinatus as found abundantly on cultivated lands near Izamal, eighty miles inland on the main, and on the rocky plain south of Progreso. I am strongly inclined to judge the latter species to be its parentage. The fruiting characters of the two species are here illustrated in conjunction. The species differ in the size of inflorescence; number and size of the involucres; character of the basal bristles (barbellate in

${ }^{*}$ Reported as C. tribuloides L. in Field Mus. Bot. $2: 44$.

$\dagger$ The specimen from Cozumel reported as this species under the description of the type proves to be $\mathrm{C}$. carolinianus Walt. 
C. echinatus, glabrous in C. insularis); and in the shape and size as well as the nervation of the first glume.

The plant is dispersionally avevectent, the burs attaching themselves by their sharp, barbed bristles to the webbed feet of swimming birds during their walks along the strand.

15. Philoxerus vermicularis (L.) R. Br.*

This species, common on the shores of the lagoon at Progreso and Silam on the Yucatan mainland, is found on the Alacrans only on a bare spot in the Sesuvium colony of Perez and Allison Islands.

From its position on the islands I am strongly inclined to judge its dispersional character to be avevectent through the agency of small migratory birds.

I6. Boerhaavia repens Linn. Sp. P1. $3 . \dagger$

This species was found only on one of the bare spots in the Sesuvium carpet of Perez Island, where it nearly covered the small area in which it had become implanted. It occurs also on the borders of the lagoon at Progreso on the main but has not been gathered at any other part of the Yucatan region.

In regard to the Alacran Reef the dispersional character of this species is avevectent, the viscous-glandular fruits attach themselves to the feet and feathers of migratory birds.

I 7. Opuntia Tuna (L.) Mill. Dict. ed. VIII:3.

Two low, spreading clumps of this cactus were observed growing from the interstices in the cairn of old coral heads thrown up in the center of the north half of Perez Island. The plants were vigorous and had apparently well adapted themselves to their environment.

Their location plainly indicates that, in this instance, the dispersional character continues to be avevectent. That some migratory bird, resting from flight upon the cairn, deposited the seed between the topmost coral heads is evident.

18. Scaevola Lobelia Murr. Linn. Syst. ed. XIII:I 78.

One individual only of this seaside species was seen upon the islets of the reef. This was growing directly under the left arm of the little wooden cross erected at the head of the grave on the north end of Perez Island. I removed a portion of this plant for herbarium purposes: this may possibly affect its continuance.

The position in which the plant grew leaves no doubt as to the avevectent character of its dispersion.

${ }^{*}$ Lithophila vermiculata Uline in Field. Mus. Bot. 2:39.

†Dr. Heimerl considers our Alacran plant to be nothing but a form transitional between the $\mathrm{B}$. viscosum and B. hirsuta of Lagasca. 


